Original Research Paper

\title{
Comparative Analysis of Skid Resistance for Different Roadway Surface Treatments
}

\author{
Osama A. Abaza, Tanay Datta Chowdhury and Mahmoud Arafat \\ Department of Civil Engineering, University of Alaska Anchorage, College of Engineering \\ 2900 Spirit Drive, EIB 301L, AK 99508, Anchorage, Alaska, USA
}

\author{
Article history \\ Received: 22-09-2017 \\ Revised: $10-10-2017$ \\ Accepted: 26-10-2017 \\ Corresponding Author: \\ Tanay Datta Chowdhury \\ Department of Civil \\ Engineering, University of \\ Alaska Anchorage, College of \\ Engineering, 2900 Spirit Drive, \\ EIB 301L, AK 99508, \\ Anchorage, Alaska, USA
}

Email: tchowdhury2@alaska.edu

\begin{abstract}
Pavement skid resistance impacts road functionality and can affect user safety and vehicle operation costs. This study was an investigation of the effect of sealcoats on skid resistance. Seven different sealcoats-fog seal, chip seal, slurry seal, sand seal, high-friction seal, cape seal, and glass seal-were lab and field tested. Laboratory samples were further tested in the field on pavement sections. To validate lab and field tests, high-friction seal was tested on recently placed pavement sections in Anchorage, Alaska. The British Pendulum tester was used to measure sealcoat skid resistance. A minimum of 40 randomized readings from the lab and field were sorted. Statistical analysis of lab and field data showed insignificant differences between all sealcoat types with the exception of cape seal. The mean skid resistance of sealcoats indicated a preference for highfriction and glass seals. On average, high-friction and glass seals had skid numbers close to 40 , providing effective skid resistance as well as safer riding quality. The field and lab data of high-friction sealcoat were validated on recently paved roads in Anchorage. The results highlighted the consistency of skid resistance measurements for the three types of testing conditions (lab, field, and real roadway) for high-friction sealcoat.
\end{abstract}

Keywords: Skid Resistance, Sealcoats, British Pendulum Tester, High Friction

\section{Introduction}

The skid resistance of highway pavements, particularly when wet, is a serious problem to highway engineers and researchers (Abdul-Malak et al., 1988). Skid resistance is "the retarding force generated by the interaction between a pavement surface and a tire under locked non-rotating conditions" (ASTM, 2013a). Improving roadway surface conditions and increasing the friction force between vehicle tires and pavement are of great concern to all transportation agencies. Unfortunately, pavement surfaces that are economically feasible to construct lose their initial frictional resistance with exposure to traffic. In addition, while the frictional resistance of dry pavement is generally good and nearly independent of speed, wet pavements often have poor frictional resistance even at low speeds (Abdul-Malak et al., 1988).

As higher-volume roads quickly reach to their terminal serviceability, it becomes increasingly important to ensure skid-resistant roadways and greater pavement service life in a cost-effective manner. Improvements in areas such as construction of frictional resistant pavement would contribute much to safer highways. Sealcoats are one of many techniques commonly used for asphalt pavement preservation. Sealcoats benefit pavement surfaces by water proofing, delaying aging due to environmental impacts (Minnesota TRS, 2016) and enhancing skid resistance.

\section{Research Objectives}

The overall objective of this study was to investigate sealcoats and conduct a comparative analysis of different sealcoats and their performance in pavement skid resistance. This study was carried out in three phases. The first phase involved laboratory simulation of different pavement surface treatments and measurements of skid resistance for the sealcoats tested. The second phase included a statistical comparative analysis of measurements for the same surface treatment sections built in the field. Lastly, validating the results found from previous two phase with the real roadway condition for a particular seal coat.

The successful completion of this research project is expected to provide useful information for design 
engineers when they are selecting pavement preservation techniques to meet system needs and performance criteria. This research will provide a useful proxy for obtaining sealcoat skid performance data. The data will help in the goal to reduce fatalities and crashes on roadways by improving the mobility and traction of vehicles.

\section{Literature Review}

\section{Background}

Sealcoats are pavement preservation treatments; they restore pavement surface conditions and protect the underlying pavement. These benefits can lead to great cost savings (Minnesota TRS, 2016). Sealcoat use is an economical method of pavement rehabilitation; it involves the application of asphalt and aggregate to an existing bituminous surface. In addition, for protecting and preserving the pavement structure, sealcoats improve the frictional resistance of highway bituminous pavements (Epps et al., 1981). Sealcoats may also be used to enrich a raveled surface, increase pavement visibility at night, reduce tire noise, improve traffic lane demarcation, and attain a uniform surface appearance (Epps et al., 1981).

As part of this research, a literature review was conducted to locate published research/technical reports that would help in determining the final methodology used. The literature review provided information on the following topics:

- Different parameters of skid resistance

- Impacts of different sealcoats on pavement skid resistance

- Critical values of skid numbers

- Environmental impacts on sealcoats

- Effect of aggregate seals on improving skid resistance

- Pavement skid resistance and safety

- General and economic benefits of sealcoats
- Nature of impacts of different sealcoats on skid resistance

- Safety benefits that result from sealcoat use

\section{General Benefits of Sealcoats}

A published study by the Washington Department of Transportation (WDOT, 2003) explained that sealcoats provide many benefits to pavement structure by delaying or eliminating further aging due to water and sun. Sealcoats help change road texture to reduce skidding, and they give the pavement a smoother uniform appearance. Though, sealcoats supply minimal additional strength to the pavement, they provide a moisture resistant layer. In addition, sealcoats provide better resistance to studded tire wear. Sealcoats can correct some existing pavement problems by waterproofing openings, enriching under-asphalted pavement. Asphalt concrete or sealcoats perform minor leveling, sealing cracks temporarily or permanently depending on the cause.

\section{Concept of Applying Pavement Sealcoats}

An effort was made by the Minnesota Department of Transportation (Minnesota TRS 1602, 2016) to explain the underlying concept behind pavement seals application (Fig. 1). The key to an effective pavement preservation application is the optimal timing of application as well as correct treatment selection. The curve shown in Fig. 1 illustrates that preventive maintenance should take place early in pavement life. Pavement preservation options include spray-applied treatments like fog seal, chip seal, slurry seal, and micro surfacing. If the trigger condition is set too low, preventive maintenance may be ineffective, as sealcoats do not contribute structural capacity to roadways. When pavement reaches poor condition, rehabilitation such as mill and fill or reconstruction is typically more cost-effective.

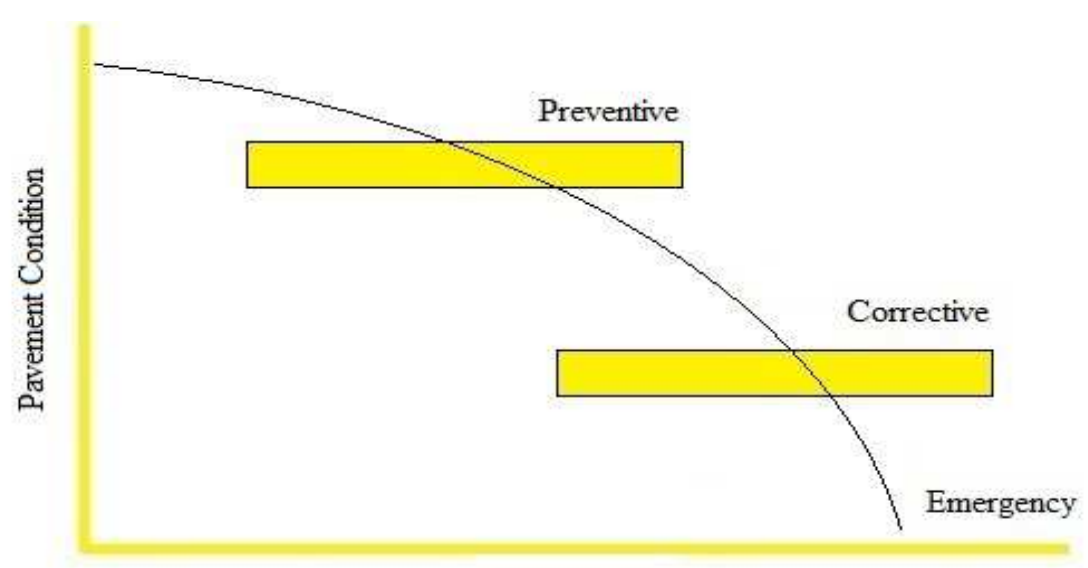

Time or Traffic

Fig. 1. Concept of applying pavement preservations (Minnesota TRS, 2016) 


\section{Parameters of Skid Resistance}

Sealcoats change the magnitude of skid resistance developed between tires and pavement surface, because skidding is generally controlled by the characteristics of the pavement surface. It can be explained by the behavior of the rubber as it rolls over the pavement surface (Abdul-Malak et al., 1988). Among the many factors that affect the role of frictional components, the most important are the micro-texture and macro-texture of pavement surface (Abdul-Malak et al., 1988). In sealcoats, micro-texture is the parameter to be considered in skid resistance and can be measured in the laboratory by using a British pendulum tester. Macro-texture is the large-scale texture of the pavement surface caused by the size and shape of the coarse aggregate particles (Abdul-Malak et al., 1988), which is outside the scope of this study.

When a survey of several states was conducted by the National Cooperative Highway Research Program (NCHRP), the consensus was that a skid number equal to or greater than 40 provides adequate surface characteristics for normal wet-weather driving conditions. Skid numbers below 40 indicate roads that need further study or corrective action to improve skid resistance (Halstead et al., 1983). NCHRP Report 37 suggests that a skid number of 37 is the minimum acceptable (Kummer and Meyer, 1967). Most state departments of transportation have established their own minimum skid number requirements, usually between 35 and 45. The Utah Department of Transportation (UDOT) uses a value of 35 as its critical skid number for designating sub-standard roads; skid numbers between 35 and 45 are considered marginal (Kummer and Meyer, 1967).

\section{Contribution of Sealcoats to Pavement Skid Resistance}

A study by the Joint Transportation Research Program (Indiana Department of Transportation and Purdue University) investigated the effect of fog seal on road surface friction (Li et al., 2012). According to this research, skid resistance measurement decreased dramatically after the application of fog seal. The average skid numbers (see Table 1) fell from 61 and 58 to 28 and 23, respectively, after application of fog seal. These numbers show that fog seal significantly reduces surface friction. The reduction of deviation of friction measurements, however, shows that fog sealing leads to a more uniform pavement surface. The study by Li et al. (2012) also shows that it normally takes the pavement surface friction about 18 months after a fog seal application to return into the original level of friction.

In a study for Florida International University (Ali and Mohammadafzali, 2014), 19 transportation agencies from Florida, Georgia, Louisiana, North Carolina, Virginia, West Virginia and Arkansas were surveyed.
Table 1. Before and after results of fog seal application (from Li et al., 2012)

\begin{tabular}{|c|c|c|c|}
\hline \multirow{2}{*}{$\begin{array}{l}\text { Before } \\
\text { Average }\end{array}$} & \multicolumn{2}{|c|}{ After } & \multirow[b]{2}{*}{ Standard Dev. } \\
\hline & Standard Dev. & Average & \\
\hline 61 & 10.8 & 28 & 4.0 \\
\hline 58 & 9.9 & 23 & 3.5 \\
\hline
\end{tabular}

Results show that $80 \%$ of the U.S. transportation agencies surveyed consider skid resistance improvement an objective of chip seal construction. Also, all agencies use chip seal to delay deterioration of the pavement and reduce water infiltration (see Fig. 2).

Based on the Florida International University survey results, slurry seal is an effective way to improve skid resistance, seal minor cracks, and waterproof the pavement surface. However, slurry seal is not a popular skid resistant sealcoat. Just two transportation agencies in Florida and Virginia reported using it (Abdul-Malak et al., 1988). Ten of the surveyed transportation agencies suggest that slurry seal has been generally replaced by micro-surfacing, which provides $45 \%$ skid resistance improvement.

\section{Environmental Impacts during Field Construction of Sealcoats}

A report by Epps et al. (1981) entitled "Field manual on design and construction of sealcoats" describes the environmental impacts taken into consideration during construction of sealcoats. Experience shows that the ideal environment for the construction of sealcoats is hot, dry weather with no rain for the next several days. Thus, the three most important environmental factors are temperature, moisture, and wind. Both road surface and atmospheric temperatures are important because they influence how well the cover aggregate can be embedded in the binder. Moreover, wet aggregate will not adhere to asphalt cement. If wet aggregate and asphalt cement are to be used successfully, they should be used on hot, low-humidity days. An excess of moisture may slow the emulsion break. The problems with moisture are reduced considerably if cationic asphalt emulsions are used. Wind speed is also a consideration. A light breeze may help evaporate moisture (or the solvent from cutbacks). In some areas, dust carried by high winds will have detrimental effects as well. If these limits are carefully observed, the chance of successfully placing a sealcoat is greatly improved.

\section{Improving Skid Resistance}

Several corrective actions can be taken to improve skid resistance on pavement sections. On low-volume roads, the UDOT uses aggregate sealcoats. 


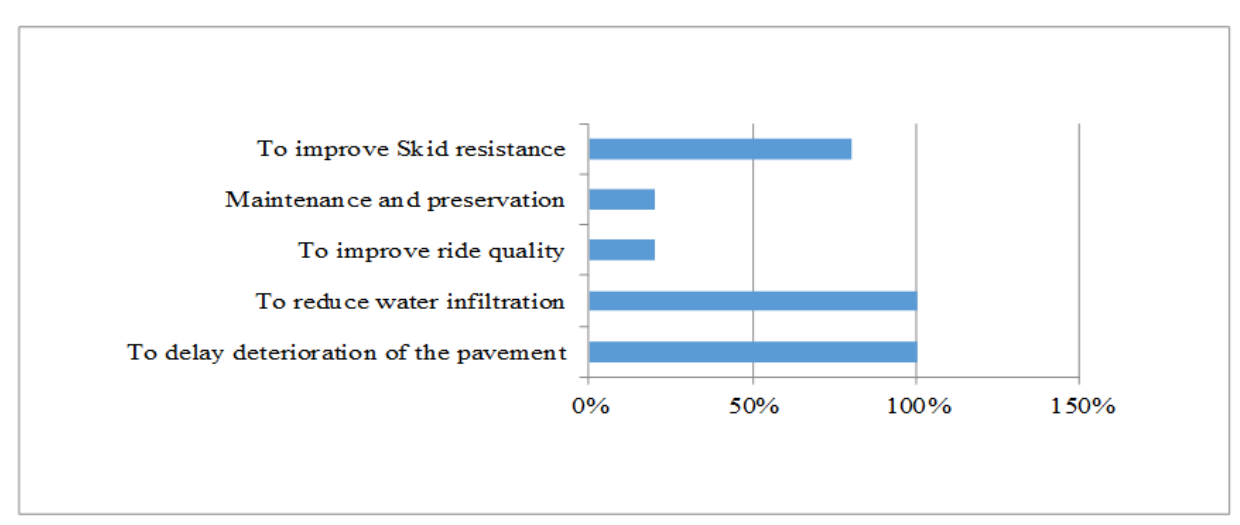

Fig. 2. Chip seal construction objectives (Ali and Mohammadafzali, 2014)

An aggregate sealcoat or "chip seal" increases road way skid resistance by replacing the polished aggregate of the original roadway with new, rough-textured aggregate particles (Blackburn et al., 1978). Aggregates used in sealcoats must meet certain criteria; they must be resistant to abrasion and polishing and must be clean and relatively free of fine materials. As long as these criteria are met, almost any type of aggregate can be used successfully in sealcoat projects (Seneviratne and Bergener, 1994).

\section{Impacts of Skid Resistance on Safety}

Driver safety is significant when designing roadway/transportation systems. Pavement skid resistance is the most important factor in reducing traffic accidents, especially in wet conditions (Mataei et al., 2016). Skid resistance is related to many factors and is a function of pavement construction materials, pavement roughness, and surface conditions (Mataei et al., 2016). Pavement skid resistance and rate of accidents show a high correlation, which requires examining the pavement friction under different conditions. Pavements must be tested in the field after they have been constructed. The British Pendulum Tester (BPT) is a low-speed, pendulum type, inexpensive, simple, and easy to use. Experience has shown that although this tester measurement is largely influenced by the micro-texture of the pavement surface, the macro-texture also affects measurements (Mataei et al., 2016). The BPT is a highly suitable tester for laboratory and field tests, as it is able to measure both longitudinal and lateral friction. This ability enables the use of the BPT to provide quality results.

\section{Economic Benefits of Sealcoats}

Sealcoats can be less expensive than continual maintenance of a gravel road, and they can be an interim step to asphalt pavement. Sealcoats economically prolong the life of existing pavement (WDOT, 2003).

A report prepared for the Utah Department of Transportation, entitled "Life Cycle of Pavement
Preservation Seal Coats" (Romero and Anderson, 2005), analyzed the performance of surface treatments on Utah pavements. Results indicated that Open-Graded Surface Courses (OGSC) have an average life, based on skid resistance, of almost 9 years and Chip Seal Courses (CSC) have a significantly longer life. Of all the factors analyzed, traffic had the most significant effect on the performance of the treatment. Based on the relative cost of both treatments and the performance observed, this study recommended that UDOT expand the use of CSC to roads with Annual Average Daily Traffic (AADT) up to 20,000 vehicles, and continue the existing procedure of using CSC in highway sections with AADT below 5,000 . The study also recommended that UDOT modify its existing policies and limit the use of OGSC where running speeds are $55 \mathrm{mph}$ or greater and AADT is in excess of 25,000 vehicles. Medium-volume facilities $(5,000$ to 25,000 AADT) should be sealed with treatments which is new to UDOT, but proven in other states as well. An initial cost analysis showed that the implementation of the changes suggested in this report should result in savings of over \$2 million per year in the agency's maintenance budget, thus allowing for better use of resources while still serving the traveling public.

\section{Methodology}

\section{Phase (I) laboratory Construction of Sealcoats and Measurements}

Seven sealcoats-chip seal, cape seal, fog seal, sand seal, slurry seal, high-friction surface treatment, and glass seal-were the focus for this study's comparison of skid resistance. Each sealcoat was applied to a $20 \mathrm{inch} \times 20 \mathrm{inch}$ flat section of plywood surface (see Fig. 3), considered the full depth pavement section.

The whole working principle was divided into two categories; one dealt with sealcoat preparation and materials used where the other dealt with using the portable BPT equipment according to the research requirement. 


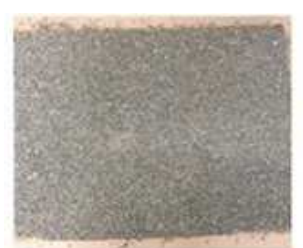

Chip Seal

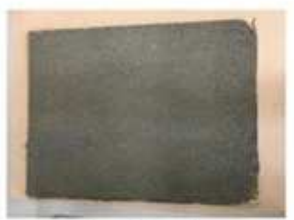

Sand Seal

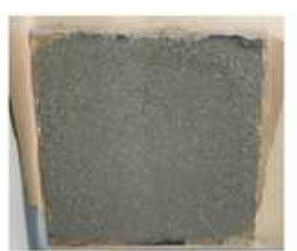

Slurry Seal

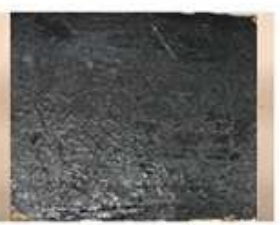

Fog Seal

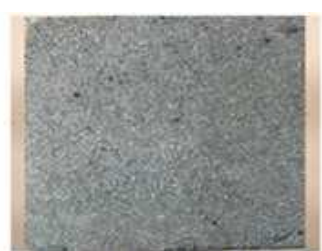

Cape Seal

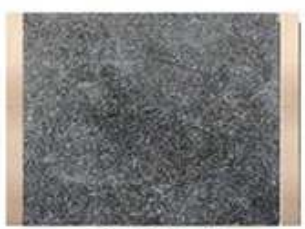

HFST Seal

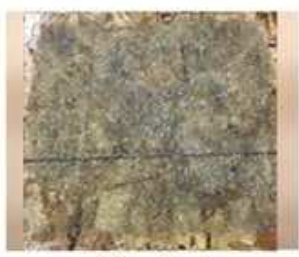

Glass Seal

Fig. 3. Samples of laboratory-simulated sealcoats

The necessary amount of material calculation for application of each sealcoat was based on the flat plywood used in this research.

\section{Phase (II) Field Construction of Sealcoats and Measurements}

An open hot mix asphalt (HMA) surface field location was designated at the University of Alaska Anchorage for conducting the test on the same seven sealcoats tested in the lab. The sealcoats were applied on a 20 inch $\times 20$ inch pavement section. The sealcoats and materials used were constructed in the field.

\section{Statistical Comparison of Skid Numbers}

A simple t-test was conducted between skid numbers from the laboratory and field results to compare the differences in skid resistance. The results from this analysis would highlight any differences between the laboratory and field results. The hypothesis testing of the results highlighted (Kutner et al., 2004):

Ho: There is no difference between the laboratory and field results (null hypothesis)

Ha: There exists significant difference between the laboratory and field results (alternative hypothesis)

The t-statistics, significance, and difference between means, standard deviation, and confidence interval were drawn from the test to identify the extent of difference between the two results. In addition, based on this statistical analysis, higher skid resistance treatment could be selected among all.

\section{Phase (III) Comparison of Skid Numbers between} Field and Actual Roadway

After selecting the best-suited sealcoats from the previous analysis, field results were compared with real roadway sealcoat results to validate the skid numbers found in both the laboratory and field. A simple $t$-test is a key to determine the real roadway condition skid numbers used with the BPT.

\section{Sealcoat Preparation and Material Description}

\section{Chip Seal}

According to AASHTO (2002), chip seal construction follows the industry standard, which consists of spreading the aggregate or chips (up to $3 / 8$ inch) onto the heated liquid bitumen over the pavement surface. In this research, $11 \mathrm{lb}$ of aggregate (3/8 in. chip) was spread over 0.16 gal of bitumen.

\section{Slurry Seal}

According to the International Slurry Surfacing Association (ISSA, 2012), slurry seal construction consists of preparing the slurry mix by mixing approved asphalt emulsions with mineral aggregate. Five pounds of slurry mix was prepared by mixing $1.1 \mathrm{lbs}$ of heated emulsions with $3.9 \mathrm{lbs}$ of aggregate (type II). Hand spreaders or squeegees were used for applying the seal over the section of clean surface plywood. 


\section{Cape Seal}

Cape seal is constructed by applying slurry seal over chip seal. Twenty-four hours was provided for drying the chip seal before applying the slurry seal on top. The aforementioned materials and method of working procedures for both seals were followed for the preparation of cape seal.

\section{Sand Seal}

Based on AASHTO (2015), the aggregate specification and the sand sealcoat preparation consisted of the application of $8 \mathrm{lbs}$. of clean sand over 0.09 gals of bitumen.

\section{Fog Seal}

Fog seal was constructed by spreading a thin coat of diluted bitumen $(0.07$ gals $)$ over the test section according to the California Department of Transportation (2003). Dilution of bitumen with water was originally recommended at $43 \%$. According to the State of California Department of Transportation (2003), however, field application of dilution is generally conducted at a rate of $50 \%$ (equal parts of emulsion and water means $1: 1$ ). The $50 \%$ rate of dilution was taken into account for this research work. The dilution water used was potable and free from any detectable solids or incompatible soluble salts to avoid any incompatible water treatment procedure.

\section{High-Friction Surface Treatment}

Based on AASHTO (2015), the aggregate specification and preparation of high-friction surface treatment (HFST) consists of spreading 0.1 gals of KwikBond HFST resin followed by immediate application of $6 \mathrm{lbs}$ of calcium bauxite. Sweeping was done on the new surface to remove excess aggregate after the resin reached its fully cured condition.

\section{Glass Seal}

A new trial sealcoat for pavements, glass chip seal, was tested. Glass seal preparation follows the same manufacturing and specification techniques as HFST. The only modification is the use of glass as the aggregate instead of traditional calcium bauxite.

Utilizing glass material in pavement structure produces contradictory results. In early research work (Korth, 1971), it was concluded that asphaltic pavements with glass aggregate provide less skid resistance than pavements with sand or similar aggregate materials. Recently, however, the addition of glass was tried again in the form of seal coating, or replacing aggregates in sealcoat materials. The company A-1 Seal coating replaces sand particles in seal coating material withecofriendly glass material, not only for solid waste management but also to improve the performance of pavements (A-1 Sealcoating, 2014). The company provided the guidelines for introducing \#80 sieve size of glass for new pavements and \#10 \#35 for maintenance work (A-1 Sealcoating, 2014).A sealcoat of glass from crushed bottles and jars combined with sealer, water, and additives was applied at a roadway for measuring performance (LaCoe, 2014). The crushed glass used for application as sealcoat ranged from \#20 to \#70 for better performance (LaCoe, 2014).

\section{British Pendulum Test}

According to ASTM (2013b), the British pendulum tester (see Fig. 4) is a dynamic impact tester used to measure energy loss when a rubber slider slides along a pavement section. The quantity measured with the portable pendulum is termed "skid resistance" or "skid number." This particular number is used to correlate with the performance of a vehicle traveling at about $10 \mathrm{~km} / \mathrm{hr}$ in real conditions on a roadway. The advantage of this particular tester is that it can be used in the field as well as in the laboratory.

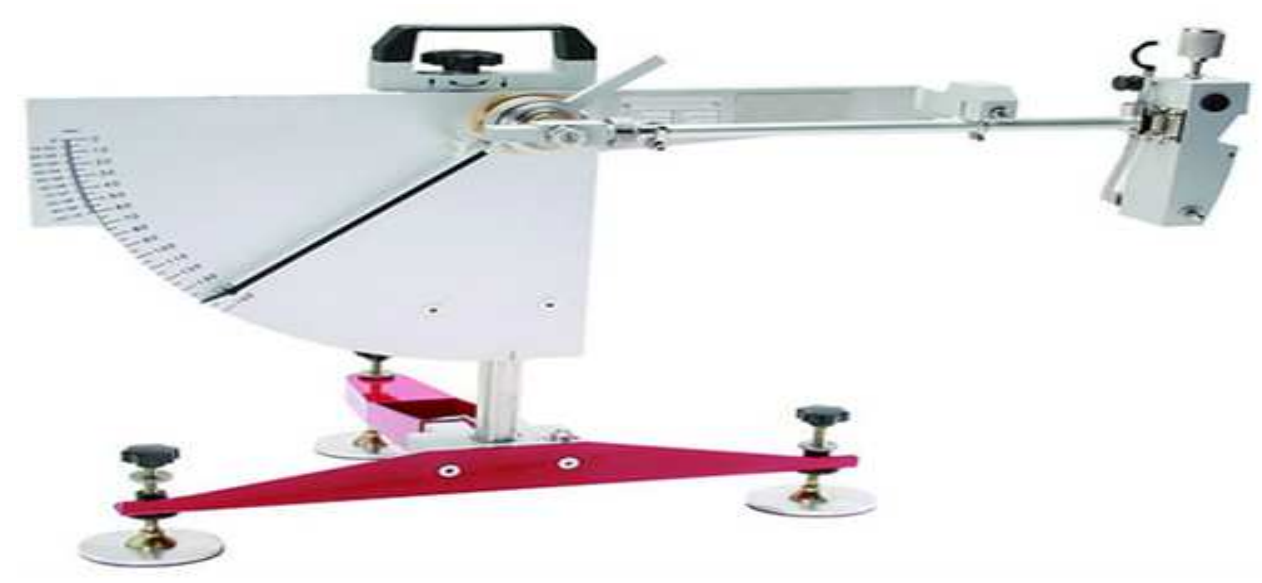

Fig. 4. British Pendulum Tester (BPT) 


\section{Test Methods}

\section{Laboratory, Field and Real Roadway Test Methods}

The research focused on measuring the skid number of different types of sealcoats in a laboratory environment as well as in the field. The sealcoats were constructed on $20 \times 20$ inch sections of flat plywood, which provides support similar to pavement structure for laboratory experiments. The same measurements were considered for the field investigation. To cover as much area for running the BPT, selections of random areas over sealcoats were considered. Forty runs of BPT were conducted over each of the sealcoats produced. Before running the BPT, the surface was properly wet and prevailing wet condition per test specifications. Repetition of the test on a single place was strictly avoided to get the best possible results. Leveling tools were used to make the surface even and proper sweeping of the surface was conducted after the curing period.A 24-hour curing period was taken into account before running the BPT over the surfaces. The selected road surface in the real roadway was also tested using BPT, applying all the guidelines mentioned above.

\section{Results and Discussion}

Forty runs of BPT over the test sections were conducted for both laboratory, field and roadway sections. Preparation of cape seal was identified as tough and difficult due to placing one seal over another, but an even surface was achieved for running the BPT. A simple t-test was performed to observe the deviation between the field and laboratory results. The results of paired difference between laboratory and field skid numbers for each surface treatment are presented in Table 2.

The mean of the differences suggest the deviation of mean between lab and field results for each of the sealcoats (Table 2). The results suggest that field results of fog, sand, and slurry seal are higher than the lab results, as mean difference suggests a negative sign. Otherwise, all lab results proved superior (meaning the lab results were higher than the field results) to the field results for the rest of the sealcoats. The differences in the mean are quite small for all seals except cape seal, ranging from -1.5 to 2.4 . The lower value of the $t$ - statistics, the higher the significance or p-value $(>0.05)$, suggesting the rejection of the alternative hypothesis (results were significantly different from each other) (Kutner et al., 2004). The standard deviation suggests the spreading of the data from the mean, and in this research, higher spreading was observed for the data obtained from sand, cape, chip, and high-friction sealcoats. The p-value of five sealcoats (fog, sand, chip, slurry, and glass) suggested the field and laboratory results are not different from each other as alternative hypothesis is rejected. Though, there is a statistically significant difference between the lab and field results of high-friction seal having p-value of 0.047 close to 0.05 . It can be considered not statistically significant as well, as field and lab results were quite close to each other. For cape seal, the t-statistics possess a higher number, and the pvalue is less than 0 , suggesting the existence of significant difference between the lab and field results. The difference of mean and the $95 \%$ confidence interval of cape seal reveal that the deviation of 8 in mean skid number between lab and field results within an interval of 6.2 to 9.8. Thus, the lab results for each sealcoat except cape seal are identical to field results in terms of skid numbers.

The skid number is an important parameter in determining the efficiency of sealcoats. The recommended guideline provided in Table 3 provides more information for selecting a specific sealcoat. After considering the guidelines in Table 3, the sealcoat that provides skid number greater than 34 should be acceptable for heavy traffic conditions. Comparing the guidelines with the results found in this research should be an effective way of selecting appropriate sealcoats.

Figure 5, presents the mean skid numbers from lab and field tests, and the average of both for each seal coat. Chip, high-friction, and glass seal showed average skid numbers higher than 30 among all. Glass and highfriction sealcoat performed significantly better, with mean skid number closer to 40. Fog seal performed poorest among all surface treatments used, having a mean skid number of 22. Sand, slurry, and cape seal resulted in lower mean skid numbers: 25.8, 26.4, and 26.8, respectively. Comparing the guidelines of Table 3, glass and high-friction sealcoat performance would provide better frictional solutions than the other sealcoats prepared for this research.

Table 2. Results of paired t-test

\begin{tabular}{|c|c|c|c|c|c|c|c|c|}
\hline \multirow[b]{3}{*}{ Pair No. } & \multirow[b]{3}{*}{ Seal } & \multicolumn{4}{|c|}{ Paired difference } & \multirow[b]{3}{*}{$\mathrm{t}$} & \multirow[b]{3}{*}{ Df } & \multirow[b]{3}{*}{ Sig. } \\
\hline & & \multirow[b]{2}{*}{ Mean } & \multirow[b]{2}{*}{ Std. deviation } & \multicolumn{2}{|c|}{$95 \%$ confidence interval } & & & \\
\hline & & & & Lower & Upper & & & \\
\hline 1 & Fog & -0.7750 & 3.6829 & -1.9528 & 0.4028 & -1.331 & 39 & 0.191 \\
\hline 2 & Sand & -1.5250 & 5.4819 & -3.2782 & 0.2282 & -1.759 & 39 & 0.086 \\
\hline 3 & Chip & 2.3500 & 7.7046 & -0.1141 & 4.8141 & 1.929 & 39 & 0.061 \\
\hline 4 & Cape & 8.0000 & 5.7065 & 6.1749 & 9.8250 & 8.866 & 39 & 0.000 \\
\hline 5 & Slurry & -1.2000 & 4.7566 & -2.7213 & 0.3213 & -1.596 & 39 & 0.119 \\
\hline 6 & High friction & 2.3250 & 7.1661 & 0.0332 & 4.6168 & 2.052 & 39 & 0.047 \\
\hline 7 & Glass & 1.1500 & 4.3651 & -0.2460 & 2.5460 & 1.666 & 39 & 0.104 \\
\hline
\end{tabular}




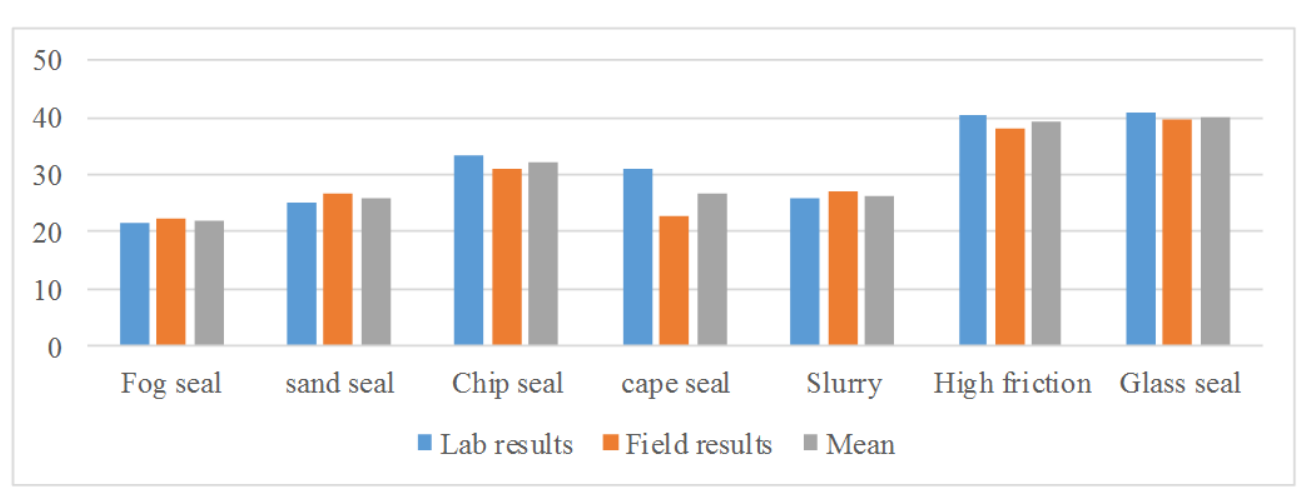

Fig. 5. Skid numbers for different sealcoats

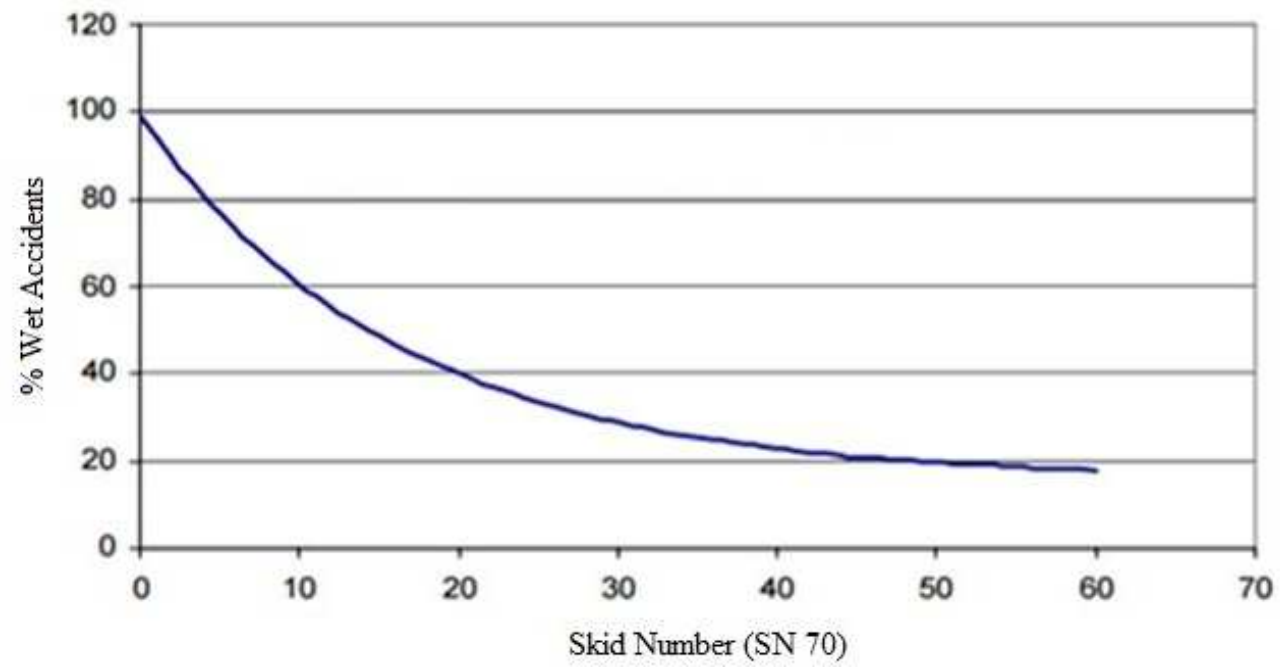

Fig. 6. Change of percentage of wet condition accidents with skid number (Source: Burchett and Rizenbergs, 1982)

Table 3. Recommended guidelines of sealcoat (After: Jayawickrama et al., 1996)

\begin{tabular}{ll}
\hline Skid number & Comments \\
\hline$<30$ & Require measures \\
$>30$ & Acceptable for roads \\
$31-34$ & Monitoring required \\
$>34$ & Acceptable for heavy traffic roads \\
\hline
\end{tabular}

A study by Burchett and Rizenbergs (1982) highlighted the influence of skid numbers in terms of wet condition accidents in Kentucky, Virginia, as presented in Fig. 6. The study revealed that skid number is highly correlated with occurrence of wet condition accidents in that particular location. The higher the skid number, the safer the drive over the pavement section, suggesting that wet condition accidents increase with decreasing skid number or skid resistance. In comparing all available sources, such as recommended guidelines and accidental influence included above on skid number, it can be concluded that pavement sections with a higher skid number provide safer driving conditions. Finally, it can be said that among the sealcoats prepared in this research, glass seal and high-friction surface treatments perform most efficiently in wet conditions.

Glass seal was found effective at providing skid resistance in roadways, along with high-friction sealcoat over this research. For validating the lab or field results to real roadway sealcoat, a high-friction sealcoat applied roadway sections was tested in Anchorage, Alaska. A section of De Armoun Road at the intersection of $\mathrm{E}$ 140th Avenue was selected for BPT result validation of the lab and field tests. As, glass is a new addition in this research, its usage is yet to be explored in a region like Alaska. A t-test was conducted of the results from the field and this location (real roadway) to identify variability (see Table 4 ). The mean difference in skid numbers between the two results indicates the real roadway condition possessed 2.25 greater mean skid numbers than the field results. Mean difference highlighting the conservative results found in the field skid resistance test compared with the real roadway condition. 


\begin{tabular}{|c|c|c|c|c|c|c|c|}
\hline \multirow[b]{3}{*}{ Pair No. High-friction Seal } & \multicolumn{7}{|c|}{ Paired difference } \\
\hline & \multirow[b]{2}{*}{ Mean } & \multirow[b]{2}{*}{ Std. deviation } & \multicolumn{2}{|c|}{$95 \%$ confidence interval } & \multirow[b]{2}{*}{$\mathrm{t}$} & \multirow[b]{2}{*}{ Df } & \multirow[b]{2}{*}{ Sig. } \\
\hline & & & Lower & Upper & & & \\
\hline Field results with real roadway & -2.25 & 11.211 & -5.84 & 1.34 & -1.269 & 39 & 0.212 \\
\hline
\end{tabular}

Both significance and $95 \%$ confidence interval, with $\mathrm{p}$ value of $0.212(p>0.05)$ and having 0 in between the confidance interval, respectively, suggest no difference between the field and real roadway condition results.

The high-friction sealcoat skid numbers in three cases (laboratory, field, and roadway) are statistically insignificant. In addition, the skid number in the field test was higher. As, validation showed positive results for high-friction sealcoat, a positive outcome is expected for the use of glass seal under the same condition of application as well. Moreover, safely laboratory experiments can be utilized for measuring skid numbers as well as building proper knowledge of skid resistance before application of any seal coats in the real roadways.

\section{Conclusion}

In this study, seven different sealcoats were tested in an attempt to increase skid resistance and improve pavement performance. Sealcoats were prepared in the laboratory setting, using flat plywood boards as a representation of the pavement section and sections of HMA in a field environment. A BPT was used to measure the skid resistance of the surface prepared with the sealcoats. Forty randomized readings in both cases (lab and field) were sorted. Statistical analysis suggests that, except for cape seal, all of the sealcoats had the same outcome in comparing lab and field data. The mean skid numbers of sealcoats showed a preference for highfriction seal and glass seal for use as sealcoat. Highfriction and glass seal provided skid numbers close to 40, indicating relatively high skid resistance, which will lead to lower crash rates as expected. Moreover, the field and lab data of high-friction sealcoat were validated on real roadway sections with high-friction sealcoats. The result highlighted the positive relation between the three types (lab, field, real roadway) of high skid number for highfriction sealcoat. As, the real roadway condition results of high friction seal coats is quietly matched with laboratory and field test, glass seal might be a promising alternative for effective sealcoat as well. Lastly, the lab simulation of sealcoat testing proved effective. In addition, high-friction and glass sealcoats are preferable for providing high skid resistance on roadways under wet conditions as well.

\section{Acknowledgment}

Special thanks to Department of Civil Engineering, University of Alaska Anchorage (UAA) for overall help and providing facilities for testing seal coats in laboratory, field and real roadway condition. All of the work was performed under the general supervision of Professor Abaza. Thank you to Matthew Iskra from Alaska Department of Transportation and Public Facilities (AKDOT\&PF) for providing the seal coat materials and guidelines for preparing the coats.

\section{Author's Contributions}

Osama A. Abaza: He was the principal investigator. Planning and organization as well as mentored the research.

Tanay Datta Chowdhury: Preparing samples (laboratory and field) and testing it in the required condition (field, laboratory and real roadway). Contributed in writing the article as well.

Mahmoud Arafat: Preparing samples (laboratory and filed) and testing it in the required conditions (field, laboratory and real roadway). Contributed in writing the article as well.

\section{Ethics}

The article is an original one. There is no ethical issue to be address for this article.

\section{References}

A-1 Sealcoating, 2014. West Fargo, North Dakota. http://a1-sealcoating.com/

AASHTO, 2002. Standard method of test for resistance to degradation of small size coarse aggregate by abrasion \& impact in Los Angeles machine. American Association of State Highway and Transportation Officials, Washington, DC.

AASHTO, 2015. Sieve analysis of fine and coarse aggregates. American Association of State Highway and Transportation Officials, Washington, DC.

Abdul-Malak, M.A.U., C.G. Papaleontiou, D.W. Fowler and A.H. Meyer, 1988. Investigation of the frictional resistance of seal coat pavement surfaces.

Ali, H. and M. Mohammadafzali, 2014. Asphalt surface treatment practice in Southeastern United States.

ASTM, 2013a. Standard terminology relating to vehiclepavement systems. American Society of Testing and Materials. 
ASTM, 2013b. Standard test method for measuring surface frictional properties using the British Pendulum Tester. American Society of Testing and Materials, Philadelphia, PA.

Blackburn, R.R., D.W. Harwood, A.D. St John and M.C. Sharp, 1978. Effectiveness of alternative skid reduction measures. Volume 1: Evaluation of Accident Rate-Skid Number Relationships (No. FHWA-RD-79-22 Final Rpt.).

Burchett, J.L. and R.L. Rizenbergs, 1982. Frictional performance of pavements and estimates of accident probability. Proceedings of the Pavement Surface Characteristics and Materials. ASTM International. DOI: $10.1520 / \mathrm{STP} 28464 \mathrm{~S}$

Epps, J.A., B.M. Gallaway and C.H. Hughes, 1981. Field manual on design and construction of sealcoats. Research Report 214-25, Texas State Department of Highways and Public Transportation.

Halstead, W.J., T.L. Copas, H.A. Pennock and A. Shipman, 1983. Criteria for use of asphalt friction surfaces. Final Report Transportation Research Board, Washington, DC.

ISSA, 2012. Asphalt slurry seal coat. International Slurry Surfacing Association Annual Meeting, pp: 1-7.

Jayawickrama, P., R. Prasanna and S. Senadheera, 1996. Survey of state practices to control skid resistance on hot-mix asphalt concrete pavements. Trans. Res. Record: J. Transportation Res. Board, 1536: 52-58. DOI: $10.3141 / 1536-08$.
Korth, M.V., 1971. The skid resistance of asphaltic pavements using glass aggregates.

Kummer, H.W. and W.E. Meyer, 1967. Tentative skidresistance requirements for main rural highways. NCHRP Report.

Kutner, M.H., C.J. Nachtsheim and J. Neter, 2004. Applied Linear Regression Models. 4th Edn., New York, McGraw-Hill.

LaCoe, R.E., 2014. Sealcoat system. US 20140161524.

Li, S., S. Noureldin, Y. Jiang and Y. Sun, 2012. Evaluation of pavement surface friction treatments.

Mataei, B., H. Zakeri, M. Zahedi and F.M. Nejad, 2016. Pavement friction and skid resistance measurement methods: A literature review. Open J. Civil Eng., 6: 37. DOI: $10.4236 /$ ojce. 2016.64046

Minnesota TRS, 2016. Local road research board. Minnesota Department of Transportation, Transportation Research Synthesis, TRS 1602-2016

Romero, P., and D. Anderson, 2005. Life cycle of pavement preservation seal coats (No. UT-04.07).

Seneviratne, P.N. and J.M. Bergener, 1994. Effects of aggregate seal coats on skid index numbers and accident rates of low volume roads in Utah (No. MPC Rept No. 94-33). Mountain-Plains Consortium.

State of California Department of Transportation, 2003. Fog seal guidelines. Caltrans Division of Maintenance, Sacramento, CA.

WDOT, 2003. Technology Transfer. Asphalt Sealcoats. Washington State Department of Transportation. 